\title{
A simple group of order 60 is isomorphic to a subgroup of $S_{6}$
}

\author{
Open Mathematics Collaboration*广
}

July 22, 2020

\begin{abstract}
We show that the permutation of six Sylow 5-subgroups by conjugation is a faithful action, so that $G$ is isomorphic to a subgroup of $S_{6}$.
\end{abstract}

keywords: permutation group, simple group, Sylow subgroup, symmetric group

The most updated version of this paper is available at https://osf.io/g43yj/download

\section{Introduction}

1. This is an open science article that aims the reproducibility of a specific result in abstract algebra in order to foster and strengthen the research in pure mathematics.

2. We present theorem (3) and prove lemma (4).

*All authors with their affiliations appear at the end of this paper.

†Corresponding author: mplobo@uft.edu.br | Join the Open Mathematics Collaboration 


\section{Theorem}

3. Any simple group $G$ of order 60 is isomorphic to $A_{5}$. [1]

\section{Lemma}

4. $G$ permutes the set $\Omega$ of six Sylow 5 -subgroups by conjugation. This action is faithful, so that $G$ is isomorphic to a subgroup of $S_{6}$.

\section{Notation \& Definition}

5. $\exists !_{2} \equiv$ exists exactly two

6. $(H \unlhd G) \equiv(H$ is a normal subgroup of $G)$

7. $(H \triangleleft G) \equiv(H$ is a proper normal subgroup of $G)$

8. $\gg$ bijection

9. $\cong$ isomorphism

10. $G \circlearrowleft A: G$ acts on $A$

11. $g \in G, \quad h \in H$

\section{Proof of Lemma (4)}

12. $G=$ simple if
(a) $G \neq\{1\}$
(b) $\exists !_{2} S \unlhd G:(S=\{1\}) \underline{\vee}(S=G)$

13. $G$ is simple.

14. $|G|=60$ 
15. $(H \unlhd G) \equiv\left(g h g^{-1} \in H\right)$

16. $G=(G, \circ)=$ group, $\quad \Omega \neq \varnothing, \quad$ points $=$ elements of $\Omega$

$\alpha \cdot g=$ rule that determines a new element of $\Omega$

$\alpha \cdot g=$ defines an action of $G$ on $\Omega(G$ acts on $\Omega)$ if

(i) $\alpha \cdot 1=\alpha, \forall \alpha \in \Omega$;

(ii) $(\alpha \cdot g) \cdot h=\alpha \cdot(g h), \forall \alpha \in \Omega, \forall g, h \in G$.

17. $\forall G, A \neq \varnothing \exists \alpha$ : (actions $G \circlearrowleft A) \nrightarrow$ (homomorphisms $f^{h}: G \rightarrow S_{A}$ )

18. $G$ permutes the set $\Omega$ of six Sylow 5 -subgroups by conjugation; $\forall H \in \Omega \forall g \in G: \alpha_{g}(H)=g H g^{-1}$.

19. Let $N=N_{G}(P)$, then $n_{p}=|G: N|$.

20. Since $n_{5}=6$, then $N$ has index 6 in $G$.

21. So the action $\alpha$ in (18) gives a permutation representation of $G$ into $S_{6}$, i.e., $\alpha$ is the homomorphism $g \mapsto \alpha_{g}$.

22. $\operatorname{ker} \alpha=\{g \in G: \alpha \cdot g=\alpha, \forall \alpha \in \Omega\} \triangleleft G$

23. From (12), (13) and (22), ker $\alpha=\{1\}$.

24. So the action is faithful.

25. Since ker $\alpha=\{1\}$, the map $G \rightarrow S_{6}$ is injective.

26. Therefore $G \cong \operatorname{Im} \alpha \leq S_{6}$.

\section{Open Invitation}

Review, add content, and co-author this paper [2,3]. Join the Open Mathematics Collaboration (https://bit.ly/ojmp-slack). Send your contribution to mplobo@uft.edu.br. 


\section{Open Science}

The latex file for this paper together with other supplementary files are available [4].

\section{Ethical conduct of research}

This original work was pre-registered under the OSF Preprints [5], please cite it accordingly [6]. This will ensure that researches are conducted with integrity and intellectual honesty at all times and by all means.

\section{Acknowledgement}

+ Center for Open Science

https://www.cos.io

+ Open Science Framework

https://osf.io

\section{References}

[1] Dummit, David Steven, and Richard M. Foote. Abstract Algebra. Vol. 3. Hoboken: Wiley, 2004.

[2] Lobo, Matheus P. "Microarticles." OSF Preprints, 28 Oct. 2019. https://doi.org/10.31219/osf.io/ejrct

[3] Lobo, Matheus P. "Simple Guidelines for Authors: Open Journal of Mathematics and Physics." OSF Preprints, 15 Nov. 2019. https://doi.org/10.31219/osf.io/fk836

[4] Lobo, Matheus P. "Open Journal of Mathematics and Physics (OJMP)." OSF, 21 Apr. 2020. https://doi.org/10.17605/osf.io/6hzyp 
[5] COS. Open Science Framework. https://osf.io

[6] Lobo, Matheus P. "A Simple Group of Order 60 Is Isomorphic to a Subgroup of S6." OSF Preprints, 24 June 2020. https://doi.org/10.31219/osf.io/g43yj

\section{The Open Mathematics Collaboration}

Matheus Pereira Lobo (lead author, mplobo@uft.edu.br) $)^{1,2}$ https://orcid.org/0000-0003-4554-1372

${ }^{1}$ Federal University of Northern Tocantins (Brazil)

${ }^{2}$ Universidade Aberta (UAb, Portugal) 